IFT-UAM/CSIC-06-49

hep-ph/0610255

\title{
Scalar Loops in Little Higgs Models
}

\author{
J.R. Espinosa and J.M. No* \\ IFT-UAM/CSIC, 28049 Madrid, Spain.
}

\begin{abstract}
Loops of the scalar particles present in Little Higgs models generate radiatively scalar operators that have been overlooked before in Little Higgs analyses. We compute them using a technique, recently proposed to deal with scalar fluctuations in non-linear sigma models, that greatly simplifies the calculation. In particular models some of these operators are not induced by loops of gauge bosons or fermions, are consistent with the Little Higgs symmetries that protect the Higgs boson mass, and must also be included in the Lagrangian. In general, scalar loops multiplicatively renormalize the tree-level scalar operators, $\mathcal{O}_{S} \rightarrow \mathcal{O}_{S}\left[1-\mathcal{N} \Lambda^{2} /(4 \pi f)^{2}\right]$ with large $\mathcal{N}$ (e.g. $\mathcal{N} \sim 20$ for the Littlest Higgs), suggesting a true UV cutoff $\Lambda \lesssim 4 \pi f / \sqrt{\mathcal{N}}$ significantly below the estimate $4 \pi f$ of naive dimensional analysis. This can have important implications for the phenomenology and viability of Little Higgs models.
\end{abstract}

October 2006

\footnotetext{
${ }^{*}$ E-mail addresses: jose.espinosa@uam.es, josemi.no@uam.es
} 


\section{Introduction}

The Little Hierarchy problem concerns the tension between the electroweak scale (determined by the Higgs mass parameter in the Lagrangian) and the $10 \mathrm{TeV}$ scale, which is roughly the minimal suppression scale of non-renormalizable operators required not to upset the fit to precision electroweak data. The little hierarchy between these two scales would require a fine-tuning of $\mathcal{O}(1 \%)$ if the low-energy effective theory below $\Lambda \sim 10 \mathrm{TeV}$ is the pure Standard Model (SM).

Little Higgs (LH) models [1-7] try to solve this little hierarchy problem by making the Higgs doublet a pseudo-Goldstone of some global symmetry $G$ which is broken (both explicitly and spontaneously) in a smart way ("collective breaking") that keeps the Higgs mass protected from 1-loop quadratically divergent corrections. Implementing such global symmetry requires new fields beyond those of the SM: new gauge bosons, fermions and scalars, filling out multiplets of $G$. The spontaneous breaking has an order parameter $f \sim 1 \mathrm{TeV}$ and the new LH particles gain masses of that order. In most existing LH models one treats this breaking in an effective way using a nonlinear sigma model description so that one keeps in the low-energy effective theory the (pseudo-)Goldstone scalars, which remain light after the spontaneous breaking of $G$. The dynamics of this breaking belongs in the complete theory at $\Lambda \sim 4 \pi f \sim 10 \mathrm{TeV}$, which acts as the limit of validity of the low-energy effective theory.

The Higgs doublet is among these pseudo-Goldstone fields but, due to the particular way in which the breaking is arranged, it does not get a mass of order $f \sim 1$ TeV but has a special protection and its mass squared is of order $f^{2} /\left(16 \pi^{2}\right)$ which, at least parametrically, is of the order of the electroweak scale, as desired. The LH scalar sector is usually treated in the following way. Calling $\Sigma$ the scalar matrix that contains the pseudo-Goldstone degrees of freedom (in some kind of non-linear exponential parametrization of fluctuations around the vacuum $\Sigma_{0}$ ) the low-energy Lagrangian for $\Sigma$ is basically determined by symmetry considerations and can be organized as a momentum expansion. The kinetic part is of the form

$$
\mathcal{L}_{k}=\frac{f^{2}}{8} \operatorname{Tr}\left[\left(D_{\mu} \Sigma\right)\left(D^{\mu} \Sigma\right)^{\dagger}\right]
$$

where $D_{\mu} \Sigma$ is the covariant derivative, which depends on the gauged subgroups of $G$ in any given model. 
The matrix $\Sigma$ also couples to fermions respecting the symmetries that protect the Higgs mass. In a generic $\Sigma$-background, fermions and gauge bosons will have $\Sigma$-dependent mass matrices $\mathcal{M}_{F}(\Sigma)$ and $\mathcal{M}_{V}(\Sigma)$. Loops of these particles will then generate scalar operators. In fact, the one-loop scalar potential contains quadratically divergent contributions of the form

$$
\delta_{q} V=\frac{\Lambda^{2}}{32 \pi^{2}} \operatorname{Str} \mathcal{M}^{2}
$$

where Str traces over degrees of freedom with negative sign for fermions. Plugging $\mathcal{M}_{F}(\Sigma)$ and $\mathcal{M}_{V}(\Sigma)$ in (2) and using the estimate of naive dimensional analysis (NDA) $\Lambda \sim 4 \pi f[8]$, one gets two types of $\Sigma$-operators:

$$
\begin{aligned}
& \mathcal{O}_{V}(\Sigma) \propto f^{2} \operatorname{Tr}\left[\mathcal{M}_{V}^{2}(\Sigma)\right], \\
& \mathcal{O}_{F}(\Sigma) \propto f^{2} \operatorname{Tr}\left[\mathcal{M}_{F}^{\dagger}(\Sigma) \mathcal{M}_{F}(\Sigma)\right],
\end{aligned}
$$

which one should include in the Lagrangian from the very beginning as they would be generated radiatively anyway. So one writes

$$
\delta_{S} \mathcal{L}=c_{V} \mathcal{O}_{V}(\Sigma)+c_{F} \mathcal{O}_{F}(\Sigma)
$$

with some unknown coefficients $c_{V}$ and $c_{F}$ expected to be $\mathcal{O}(1)$ (although in particular models these might turn out to be sizeable [9]).

In fact such operators are crucial for the viability of LH models: they generate $\mathcal{O}(f)$ masses for all pseudo-Goldstones other than the doublet Higgs, and scalar quartic couplings, including that of the Higgs doublet. In the presence of the non-derivative scalar interactions of (4) one might wonder about the scalar contribution to (2), which is missing in LH analyses in the literature. Analyzing this issue is the main motivation of this paper.

Perhaps one of the reasons for overlooking this scalar contribution is that its calculation is not as easy as that for the scalar operators induced by fermion and gauge boson loops. To compute the contribution from scalar loops one would need first to find $\mathcal{M}_{S}^{2}(\Sigma)$, the squared mass matrix for scalar fluctuations in a general $\Sigma$-background. One immediate complication is that taking derivatives of (4) with respect to scalar fields [to get $\mathcal{M}_{S}^{2}(\Sigma)$ ] destroys the $\Sigma$ structure so that it seems impossible to write down the matrix elements $\mathcal{M}_{S}^{2}(\Sigma)_{a b}$ as functions of $\Sigma$. Somehow one expects that all 
the bits and pieces in $\mathcal{M}_{S}^{2}(\Sigma)_{a b}$ will arrange themselves so as to give a $\operatorname{Tr}\left[\mathcal{M}_{S}^{2}(\Sigma)\right]$ that can indeed be expressed as a function of $\Sigma$.

To complicate things further, in a generic $\Sigma$-background the kinetic terms in (1) are not canonical. The $\Sigma$-dependent re-scaling of scalar fluctuations required to get back to canonical kinetic terms also affects the form of $\mathcal{M}_{S}^{2}(\Sigma)$ entering in (2). The programme then might be straightforward but seems rather cumbersome and tedious. Fortunately, a method recently developed in [10] to deal efficiently with scalar fluctuations in nonlinear sigma models, is ideally suited for our task.

We explain this method in the next section, using as an illustrative example the Littlest Higgs model [1], and calculate $\operatorname{Tr}\left[\mathcal{M}_{S}^{2}(\Sigma)\right]$ to see what scalar operators are generated in this way. In section 3 we confront the scalar-induced $\Sigma$-operators with the operators generated by loops of gauge bosons and fermions discussing their symmetry properties and their expansions in terms of physical fields. In section 4 we examine some of the implications of these scalar loop corrections to the effective potential. In particular, we show how the appearance of scalar-induced operators can be used to set an upper bound on the scale $\Lambda$ up to which the LH effective theory is valid. From purely low-energy arguments, it follows that such cutoff is in general significantly lower than $4 \pi f$ (e.g., it is $\sim 4 \pi f / \sqrt{20}$ for the Littlest Higgs model). This can have implications for the ability of LH models to solve the little hierarchy problem. Some implications for vacuum alignment issues are also discussed. In section 5 we present some conclusions. Appendix A is devoted to logarithmically divergent corrections from scalar loops using again as example the Littlest Higgs model. Appendix B deals with scalar loop corrections in a different LH model, based on $S U(6) / S p(6)$, as an example in which scalar-induced operators are truly new and different from those generated by loops of gauge bosons or fermions. Finally, Appendix C examines a toy $S U(N) / S O(N)$ LH model to investigate how the new bound on the UV cut-off derived in this paper scales with the size of the groups involved.

\section{An Efficient Way of Dealing with Scalar Fluctu- ations}

It is convenient to use a concrete model to illustrate the method we follow to compute in an efficient way $\operatorname{Tr}\left[\mathcal{M}_{S}^{2}(\Sigma)\right]$. We use for that purpose the original Littlest Higgs model 
$[1,11]$ even if it is difficult to reconcile with electroweak precision tests (such issues are not relevant for the method itself and we prefer to keep the model simple). The model is based on a non-linear sigma model with coset structure $S U(5) / S O(5)$. The spontaneous breaking of $S U(5)$ down to $S O(5)$ is produced by the vacuum expectation value (VEV) of a $5 \times 5$ symmetric matrix $\Psi$ [which transforms under $S U(5)$ as $\Psi \rightarrow$ $\left.U \Psi U^{T}\right]$, e.g. when $\langle\Psi\rangle=I_{5}$ (we call $I_{n}$ the $n \times n$ identity matrix). The breaking of this global $S U(5)$ symmetry produces 14 Goldstone bosons among which lives the scalar Higgs field. Following [1], we make a basis change from $\langle\Psi\rangle=I_{5}$ to $\langle\Psi\rangle=\Sigma_{0}$ where

$$
\Sigma_{0}=\left(\begin{array}{ccc}
0 & 0 & I_{2} \\
0 & 1 & 0 \\
I_{2} & 0 & 0
\end{array}\right) .
$$

Let us call $U_{0}$ the $S U(5)$ matrix that performs this change of basis. One has then $\Sigma_{0}=U_{0} U_{0}^{T}$ and all the group generators transform as $t_{a}=U_{0} t_{a}^{(0)} U_{0}^{\dagger}\left[t_{a}^{(0)}\right.$ are the generators in the original basis]. The unbroken $S O(5)$ generators satisfied the obvious relation $T_{a}^{(0)}+T_{a}^{(0) T}=0$ and multiplying on the left by $U_{0}$ and on the right by $U_{0}^{T}$ we arrive at the condition

$$
T_{a} \Sigma_{0}+\Sigma_{0} T_{a}^{T}=0
$$

for the generators in the new basis (a condition which is immediate to obtain alternatively just by requiring invariance of $\Sigma_{0}$ ). In the original basis the broken generators obviously satisfy $X_{a}^{(0)}=X_{a}^{(0) T}$. Multiplying again by $U_{0}$ and $U_{0}^{T}$ one gets in the transformed basis

$$
X_{a} \Sigma_{0}=\Sigma_{0} X_{a}^{T} .
$$

The Goldstone boson degrees of freedom can be parametrized through the nonlinear sigma model field

$$
\Sigma \equiv e^{i \Pi / f} \Sigma_{0} e^{i \Pi^{T} / f}=e^{2 i \Pi / f} \Sigma_{0},
$$

where $\Pi=\sum_{a} \pi_{a} X_{a}$ and we can choose the $\pi_{a}$ as real fields (in which case $a$ runs from 1 to 14$)$. The model has a gauged $S U(2)_{1} \times S U(2)_{2} \times U(1)_{1} \times U(1)_{2}$ subgroup of $S U(5)$ with generators

$$
Q_{1}^{\alpha}=\left(\begin{array}{cc}
\sigma^{\alpha} / 2 & \\
& 0_{3}
\end{array}\right), \quad Q_{2}^{\alpha}=\left(\begin{array}{cc}
0_{3} & \\
& -\sigma^{\alpha^{*}} / 2
\end{array}\right),
$$

(where $\sigma^{\alpha}$ are the Pauli matrices) and

$$
Y_{1}=\operatorname{diag}(-3,-3,2,2,2) / 10, \quad Y_{2}=\operatorname{diag}(-2,-2,-2,3,3) / 10 .
$$


The VEV in eq. (5) additionally breaks $S U(2)_{1} \times S U(2)_{2} \times U(1)_{1} \times U(1)_{2}$ down to the $\mathrm{SM} S U(2)_{L} \times U(1)_{Y}$ group.

The hermitian matrix $\Pi$ in eq. (8) contains the Goldstone and (pseudo)-Goldstone bosons:

$$
\Pi=\left(\begin{array}{ccc}
\xi & \frac{H^{\dagger}}{\sqrt{2}} & \Phi^{\dagger} \\
\frac{H}{\sqrt{2}} & 0 & \frac{H^{*}}{\sqrt{2}} \\
\Phi & \frac{H^{T}}{\sqrt{2}} & \xi^{T}
\end{array}\right)+\frac{1}{\sqrt{20}} \zeta^{0} \operatorname{diag}(1,1,-4,1,1)
$$

where $H=\left(h^{0}, h^{+}\right)$is the Higgs doublet; $\Phi$ is a complex $S U(2)$ triplet given by the symmetric $2 \times 2$ matrix:

$$
\Phi=\left[\begin{array}{cc}
\phi^{0} & \frac{1}{\sqrt{2}} \phi^{+} \\
\frac{1}{\sqrt{2}} \phi^{+} & \phi^{++}
\end{array}\right]
$$

the field $\zeta^{0}$ is a singlet which is the Goldstone associated to $U(1)_{1} \times U(1)_{2} \rightarrow U(1)_{Y}$ breaking and finally, $\xi$ is the real triplet of Goldstone bosons associated to $S U(2)_{1} \times$ $S U(2)_{2} \rightarrow S U(2)$ breaking:

$$
\xi=\frac{1}{2} \sigma^{\alpha} \xi^{\alpha}=\left[\begin{array}{cc}
\frac{1}{2} \xi^{0} & \frac{1}{\sqrt{2}} \xi^{+} \\
\frac{1}{\sqrt{2}} \xi^{-} & -\frac{1}{2} \xi^{0}
\end{array}\right] .
$$

The kinetic part of the Lagrangian is of the general form (1) with

$$
D_{\mu} \Sigma=\partial_{\mu} \Sigma-i \sum_{j=1}^{2} g_{j} W_{j \mu}^{\alpha}\left(Q_{j}^{\alpha} \Sigma+\Sigma Q_{j}^{\alpha T}\right)-i \sum_{j=1}^{2} g_{j}^{\prime} B_{j \mu}\left(Y_{j} \Sigma+\Sigma Y_{j}^{T}\right) .
$$

In this model, additional fermions are introduced as a vector-like coloured pair $\tilde{t}, \tilde{t}^{c}$ to cancel the quadratic divergence from top loops (we neglect the other small Yukawa couplings). The relevant part of the Lagrangian containing the top Yukawa coupling is given by

$$
\mathcal{L}_{f}=\frac{1}{2} h_{1} f \epsilon_{i j k} \epsilon_{x y} \chi_{i} \Sigma_{j x} \Sigma_{k y} u_{3}^{\prime c}+h_{2} f \tilde{t} \tilde{t}^{c}+h . c .,
$$

where $\chi_{i}=(b, t, \tilde{t})$, indices $i, j, k$ run from 1 to 3 and $x, y$ from 4 to 5 , and $\epsilon$ is the completely antisymmetric tensor.

As explained in the introduction, considering gauge and fermion loops, one sees that the Lagrangian should also include gauge invariant terms of the form,

$$
\begin{aligned}
-\mathcal{L}_{S}=V & =2 c_{V_{i}} f^{4} g_{i}^{2} \sum_{\alpha} \operatorname{Tr}\left[\left(Q_{i}^{\alpha} \Sigma\right)\left(Q_{i}^{\alpha} \Sigma\right)^{*}\right]+2 c_{Y} f^{4} g^{\prime 2} \operatorname{Tr}\left[(Y \Sigma)(Y \Sigma)^{*}\right] \\
& +4 c_{F} f^{4} h_{1}^{2} \operatorname{Tr}\left[\Sigma_{1} \epsilon \Sigma_{1}^{T} \Sigma_{1}^{*} \epsilon \Sigma_{1}^{\dagger}\right]
\end{aligned}
$$

where $\Sigma_{1}$ is a $3 \times 2$ matrix defined by

$$
\Sigma_{1} \equiv\left(\Sigma_{i x}\right)
$$


with $i=\{1,2,3\}, x=\{4,5\}$ and $\epsilon$ is the $2 \times 2$ completely antisymmetric tensor so that

$$
\operatorname{Tr}\left[\Sigma_{1} \epsilon \Sigma_{1}^{T} \Sigma_{1}^{*} \epsilon \Sigma_{1}^{\dagger}\right]=\epsilon^{w x} \epsilon_{z y} \Sigma_{i w} \Sigma_{j x} \Sigma^{i y *} \Sigma^{j z *} .
$$

Finally, $c_{V_{i}}, c_{Y}$ and $c_{F}$ are assumed to be constants of $\mathcal{O}(1)$.

This Lagrangian produces a mass of order $f$ for the gauge bosons $\left(W^{\prime}\right)$ associated to the broken (axial) $S U(2)$, for a vector-like combination of $\tilde{t}$ and $u_{3}^{\prime}$ and for the complex scalar $\Phi$. Finally, the Higgs boson has zero tree level mass but a nonzero quartic coupling.

Let us now discuss the method introduced in [10] to compute the masses of scalar fluctuations (i.e. the degrees of freedom in $\Sigma$ itself) for instance in a simple background $h=\sqrt{2}\left\langle h^{0}\right\rangle$. The standard way of doing this would be to shift $h^{0} \rightarrow h^{0}+h / \sqrt{2}$ in $\Sigma$ to compute $h$-dependent scalar masses. As explained in the Introduction, to do this properly one should take into account that in general, after this shifting, the scalar kinetic terms from (1) are not canonical. Therefore one should re-scale the fields to get the kinetic terms back to canonical form and this re-scaling affects the $h$-dependent contributions to scalar masses. Instead of following this standard procedure, ref. [10] used an alternative method which simplifies the calculations and has many appealing features.

The idea is to treat the new background with $\langle H\rangle=h / \sqrt{2}$ (or any other generic background) as a basis change (recall the discussion above of the change from $\langle\Phi\rangle=$ $I_{5}$ to $\left.\langle\Phi\rangle=\Sigma_{0}\right)$. The $S U(5)$ transformation to the new background is now $U_{b} \equiv$ $\exp (i\langle\Pi\rangle / f)$ with $\Sigma_{b} \equiv\langle\Sigma\rangle=U_{b} \Sigma_{0} U_{b}^{T}$. To parametrize the scalar fluctuations around this background one again uses the exponentials of broken generators, but taking into account the effect of the change of basis, which acts on generators as $X_{a} \rightarrow U_{b} X_{a} U_{b}^{\dagger}$. That is, instead of using $\exp (i \Pi / f)$ one uses $U_{b} \exp (i \Pi / f) U_{b}^{\dagger}$, and writes for $\Sigma$ :

$$
\Sigma=\left(U_{b} e^{i \Pi / f} U_{b}^{\dagger}\right)\left(U_{b} \Sigma_{0} U_{b}^{T}\right)\left(U_{b}^{*} e^{i \Pi^{T} / f} U_{b}^{T}\right)=e^{i\langle\Pi\rangle / f} e^{2 i \Pi / f} e^{i\langle\Pi\rangle / f} \Sigma_{0}
$$

The prescription in eq. (19), which we could call "multiplicative", is to be compared with the standard ("additive") procedure

$$
\Sigma=e^{2 i(\Pi+\langle\Pi\rangle) / f} \Sigma_{0}
$$

As already discussed in [10], it is easy to check that with the prescription of eq. (19) scalar fluctuations are automatically canonical. Second, one is free to choose this 
parametrization of scalar fluctuations: a general theorem [12] guarantees that this different parametrization does not change the physics.

The parametrization above will be most useful for our goal in this paper ${ }^{1}$. We need to compute scalar masses in a generic $\Sigma$-background. As $\operatorname{Tr}\left[\mathcal{M}^{2}(\Sigma)\right]$ is linear in $\mathcal{M}^{2}(\Sigma)$ we can compute separately the contributions to the trace coming from the scalar operators $\mathcal{O}_{V_{i}}(\Sigma), \mathcal{O}_{Y}(\Sigma)$ and $\mathcal{O}_{F}(\Sigma)$ separately. For the gauge boson induced operators we have to compute $\delta_{V} \operatorname{Tr}\left[\mathcal{M}_{S}^{2}(\Sigma)\right]$, which is given by

$$
\begin{gathered}
2 c_{V_{i}} f^{4} g_{i}^{2} \sum_{a} \operatorname{Tr}\left[\left(Q_{i}^{\alpha} \partial_{a}^{2} \Sigma\right)\left(Q_{i}^{\alpha} \Sigma\right)^{*}+2\left(Q_{i}^{\alpha} \partial_{a} \Sigma\right)\left(Q_{i}^{\alpha} \partial_{a} \Sigma\right)^{*}+\left(Q_{i}^{\alpha} \Sigma\right)\left(Q_{i}^{\alpha} \partial_{a}^{2} \Sigma\right)^{*}\right] \\
+2 c_{Y_{i}} f^{4} g_{i}^{\prime 2} \sum_{a} \operatorname{Tr}\left[\left(Y_{i} \partial_{a}^{2} \Sigma\right)\left(Y_{i} \Sigma\right)^{*}+2\left(Y_{i} \partial_{a} \Sigma\right)\left(Y_{i} \partial_{a} \Sigma\right)^{*}+\left(Y_{i} \Sigma\right)\left(Y_{i} \partial_{a}^{2} \Sigma\right)^{*}\right] .
\end{gathered}
$$

For the fermion induced scalar operators $\mathcal{O}_{F}(\Sigma)$ in the Lagrangian we have to compute

$$
\delta_{F} \operatorname{Tr}\left[\mathcal{M}_{S}^{2}(\Sigma)\right]=4 c_{F} f^{4} h_{1}^{2} \sum_{a} \partial_{a}^{2} \operatorname{Tr}\left[\Sigma_{1} \epsilon \Sigma_{1}^{T} \Sigma_{1}^{*} \epsilon \Sigma_{1}^{\dagger}\right]
$$

which we refrain from expanding further in terms of $\partial_{a} \Sigma_{1}$ and $\partial_{a}^{2} \Sigma_{1}$ because the final expression is too lengthy.

Therefore, we need to compute derivatives of $\Sigma$ with respect to the real scalar fields $\pi_{a}$ in $\Pi=\sum_{a} \pi_{a} X_{a}$, evaluated at $\pi_{a}=0$. Once we have eq. (19), such derivatives of $\Sigma$ are simple to compute. One gets

$$
\begin{aligned}
\left.\partial_{a} \Sigma\right|_{0} & =\frac{2 i}{f} e^{i\langle\Pi\rangle / f} X_{a} e^{i\langle\Pi\rangle / f} \Sigma_{0}, \\
\left.\partial_{a}^{2} \Sigma\right|_{0} & =-\frac{4}{f^{2}} e^{i\langle\Pi\rangle / f} X_{a} X_{a} e^{i\langle\Pi\rangle / f} \Sigma_{0},
\end{aligned}
$$

and their conjugates

$$
\begin{aligned}
& \left.\partial_{a} \Sigma^{*}\right|_{0}=-\frac{2 i}{f} e^{-i\left\langle\Pi^{T}\right\rangle / f} X_{a}^{*} e^{-i\left\langle\Pi^{T}\right\rangle / f} \Sigma_{0}, \\
& \left.\partial_{a}^{2} \Sigma^{*}\right|_{0}=-\frac{4}{f^{2}} e^{-i\left\langle\Pi^{T}\right\rangle / f} X_{a}^{*} X_{a}^{*} e^{-i\left\langle\Pi^{T}\right\rangle / f} \Sigma_{0} .
\end{aligned}
$$

From this point onwards we drop the brackets in $\langle\Pi\rangle$ as we are going to interpret $\operatorname{Tr}\left[\mathcal{M}^{2}(\Sigma)\right]$ as a source of new operators to be added to the Lagrangian from the beginning. To compute this trace, the second derivatives in (23) and (24) will always

\footnotetext{
${ }^{1}$ The same technique can be applied to the calculation of logarithmically divergent corrections, see Appendix A.
} 
be summed in $a$. Then notice that, using the fact that the $X_{a}$ are the broken generators, one has

$$
\sum_{a} X_{a} X_{a}=C_{S U(5)}(5)-C_{S O(5)}(5)=\frac{14}{5} I_{5},
$$

(where the $C$ 's are the quadratic Casimir operators for the fundamental representations) and so, the terms in (21) and (22) containing $\partial_{a}^{2} \Sigma$ and $\partial_{a}^{2} \Sigma_{1}$ are trivial to compute. In fact, all such terms generate operators proportional to $\mathcal{O}_{V_{i}}(\Sigma), \mathcal{O}_{Y_{i}}(\Sigma)$ or $\mathcal{O}_{F}(\Sigma)$. The rest of the terms are more interesting and a bit harder to compute. The simplest way to proceed is to notice the following two identities (derived in Appendix C), valid for generic $5 \times 5$ matrices $Y$ and $Z$ :

$$
\begin{aligned}
\sum_{a} \operatorname{Tr}\left[X_{a} Y X_{a}^{*} Z\right] & =\frac{1}{2} \operatorname{Tr}\left[Y Z^{T}\right]-\frac{1}{5} \operatorname{Tr}[Y Z]+\frac{1}{2} \operatorname{Tr}\left[Y \Sigma_{0}^{*}\right] \operatorname{Tr}\left[Z \Sigma_{0}\right] \\
\sum_{a} \operatorname{Tr}\left[X_{a} Y X_{a} Z\right] & =\frac{1}{2} \operatorname{Tr}[Y] \operatorname{Tr}[Z]-\frac{1}{5} \operatorname{Tr}[Y Z]+\frac{1}{2} \operatorname{Tr}\left[Y \Sigma_{0} Z^{T} \Sigma_{0}^{*}\right]
\end{aligned}
$$

[Note in particular that eq. (25) agrees with the second formula above, for the particular case $Y=Z=I_{5}$.] By using these identities one can easily show that $\delta_{V} \operatorname{Tr}\left[\mathcal{M}_{S}^{2}(\Sigma)\right]$ in (21) gives $\Sigma$-operators proportional to $\mathcal{O}_{V_{i}}(\Sigma)$ and $\mathcal{O}_{Y_{i}}(\Sigma)$ or field-independent. Most of the contributions from $\delta_{F} \operatorname{Tr}\left[\mathcal{M}_{S}^{2}(\Sigma)\right]$ in $(22)$ are also proportional to $\mathcal{O}_{F}(\Sigma)$ but a few give a different operator. These are

$$
4 c_{F} f^{4} h_{1}^{2} \sum_{a} \operatorname{Tr}\left[\partial_{a} \Sigma_{1} \epsilon \Sigma_{1}^{T}\left(\partial_{a} \Sigma_{1}^{*} \epsilon \Sigma_{1}^{\dagger}+\Sigma_{1}^{*} \epsilon \partial_{a} \Sigma_{1}^{\dagger}\right)\right]+\text { h.c. }
$$

which produce terms proportional to

$$
\mathcal{O}_{S}(\Sigma) \equiv \operatorname{Tr}\left[\Sigma_{1}^{\dagger} \Sigma_{1}\right]=\Sigma_{i x} \Sigma^{i x^{*}}
$$

This looks like a new type of operator one has to add to the Littlest Higgs Lagrangian from the start.

Before analyzing $\mathcal{O}_{S}(\Sigma)$ by expanding it in powers of the physical fields $H$ and $\Phi$ (which we leave for the next section) we have to discuss the following two issues. The first is to calculate the contribution of $\mathcal{O}_{S}(\Sigma)$ itself to $\operatorname{Tr}\left[\mathcal{M}^{2}(\Sigma)\right]$. It is in fact quite easy to see that such contribution,

$$
\delta_{S} \operatorname{Tr}\left[\mathcal{M}_{S}^{2}(\Sigma)\right] \propto \sum_{a} \operatorname{Tr}\left[\left(\partial_{a}^{2} \Sigma_{1}\right) \Sigma_{1}^{\dagger}+2\left(\partial_{a} \Sigma_{1}\right)\left(\partial_{a} \Sigma_{1}^{\dagger}\right)+\Sigma_{1}\left(\partial_{a}^{2} \Sigma_{1}^{\dagger}\right)\right]
$$

is proportional to $\mathcal{O}_{S}(\Sigma)$ so that no other new operators are generated and we can stop at this point. The second issue is to check that $\mathcal{O}_{S}(\Sigma)$ does indeed respect the 
symmetries of the low-energy effective theory, in particular the $S U(3)_{i}$ symmetries that are all-important to guarantee the lightness of the Higgs. We do this in the following section.

\section{Scalar Operators Revisited}

When $g_{1}=g_{1}^{\prime}=0$ the Lagrangian is invariant under $S U(3)_{1} \times S U(2)_{2}$ with $S U(3)_{1}$ global and $S U(2)_{2}$ local, with $\Sigma \rightarrow U \Sigma U^{T}$ and

$$
U=\left(\begin{array}{cc}
U_{1} & 0 \\
0 & V_{2}
\end{array}\right),
$$

where $U_{1}$ is a $3 \times 3 S U(3)$ matrix and $V_{2}$ a $2 \times 2 S U(2)$ matrix. We will call this a type-1 symmetry transformation. Under the global $S U(3)_{1}$, the Higgs shifts by a constant and this forbids a mass term for it. When $g_{2}=g_{2}^{\prime}=0$ the Lagrangian is invariant under $S U(2)_{1} \times S U(3)_{2}$ with $S U(3)_{2}$ global and $S U(2)_{1}$ local, as before, but with

$$
U=\left(\begin{array}{cc}
V_{1} & 0 \\
0 & U_{2}
\end{array}\right)
$$

where $U_{2}$ is a $3 \times 3 S U(3)$ matrix and $V_{1}$ a $2 \times 2 S U(2)$ matrix (type- 2 symmetry transformation). Again, invariance under the global $S U(3)_{2}$ forbids a mass term for the Higgs. Generating a non-zero Higgs mass requires both type- 1 and type- 2 couplings simultaneously and quadratically divergent one-loop radiative corrections do not generate such mass term (only the softer logarithmically divergent corrections can generate it).

Under type-1 symmetry transformations (30), $\Sigma_{1}$ transforms simply as

$$
\Sigma_{1} \rightarrow U_{1} \Sigma_{1} V_{2}^{T}
$$

and $\operatorname{Tr}\left[\Sigma_{1}^{\dagger} \Sigma_{1}\right]$ is obviously invariant. Under the type-2 transformations, (31), $\Sigma_{1}$ does not transform in a simple way under $S U(3)_{2}$ and $\operatorname{Tr}\left[\Sigma_{1}^{\dagger} \Sigma_{1}\right]$ is not invariant under that global symmetry. Of course invariance under the local $S U(2)_{1}$ is still maintained $\left[S U(2)_{1}\right.$ is in fact a subgroup of $\left.S U(3)_{1}\right]$.

After this simple discussion several facts become obvious. First, using $\Sigma_{1}$ it is straightforward to construct other operators that are type-1 invariant, for instance

$$
\mathcal{O}_{n} \equiv \operatorname{Tr}\left[\left(\Sigma_{1}^{\dagger} \Sigma_{1}\right)^{n}\right]
$$


In addition to these, one can make use of the $2 \times 2$ antisymmetric tensor $\epsilon$ to get new $S U(2)$ invariant combinations and write type-1 operators like

$$
\mathcal{O}_{n}^{\epsilon} \equiv \operatorname{Tr}\left[\left(\Sigma_{1} \epsilon \Sigma_{1}^{T} \Sigma_{1}^{*} \epsilon \Sigma_{1}^{\dagger}\right)^{n}\right]
$$

For $n=1$ this corresponds to the scalar operator induced by fermion loops. Other combinations give mixed operators like

$$
\mathcal{O}_{m} \equiv \operatorname{Tr}\left[\left(\Sigma_{1} \epsilon \Sigma_{1}^{T}\right)\left(\Sigma_{1}^{*} \Sigma_{1}^{T}\right)\left(\Sigma_{1}^{*} \epsilon \Sigma_{1}^{\dagger}\right)\right]
$$

and so on.

Finally, it is clear that type- 1 and type-2 symmetry transformations are quite similar and therefore one can define another $3 \times 2$ submatrix

$$
\Sigma_{2}=\left(\Sigma_{\hat{i} \hat{x}}\right)
$$

with $\hat{i}=\{3,4,5\}$ and $\hat{x}=\{1,2\}$. Under type- 2 symmetry transformations we have

$$
\Sigma_{2} \rightarrow U_{2} \Sigma_{2} V_{1}^{T}
$$

in such a way that operators invariant under type-2 transformations are trivial to construct, simply replacing $\Sigma_{1} \rightarrow \Sigma_{2}$ in all the operators above.

The scalar operators induced by loops of gauge bosons,

$$
\begin{aligned}
& \mathcal{O}_{V_{i}}(\Sigma) \equiv \sum_{\alpha} \operatorname{Tr}\left[\left(Q_{i}^{\alpha} \Sigma\right)\left(Q_{i}^{\alpha} \Sigma\right)^{*}\right] \\
& \mathcal{O}_{Y_{i}}(\Sigma) \equiv \operatorname{Tr}\left[\left(Y_{i} \Sigma\right)\left(Y_{i} \Sigma\right)^{*}\right]
\end{aligned}
$$

can in fact be rewritten in a simpler way in terms of the former operators for $\Sigma_{1}$ and $\Sigma_{2}$. One gets

$$
\begin{aligned}
& \mathcal{O}_{V_{1}}(\Sigma)=\frac{1}{2}-\frac{1}{4} \operatorname{Tr}\left[\Sigma_{2}^{\dagger} \Sigma_{2}\right] \\
& \mathcal{O}_{Y_{1}}(\Sigma)=\frac{3}{10}-\frac{1}{4} \operatorname{Tr}\left[\Sigma_{2}^{\dagger} \Sigma_{2}\right],
\end{aligned}
$$

and

$$
\begin{aligned}
& \mathcal{O}_{V_{2}}(\Sigma)=\frac{1}{2}-\frac{1}{4} \operatorname{Tr}\left[\Sigma_{1}^{\dagger} \Sigma_{1}\right] \\
& \mathcal{O}_{Y_{2}}(\Sigma)=\frac{3}{10}-\frac{1}{4} \operatorname{Tr}\left[\Sigma_{1}^{\dagger} \Sigma_{1}\right]
\end{aligned}
$$


The scalar operator induced by fermion loops, $\mathcal{O}_{F}(\Sigma)=\mathcal{O}_{1}^{\epsilon}$, see $(34)$, can be written simply as

$$
\mathcal{O}_{F}(\Sigma)=-2 \operatorname{Det}\left[\Sigma_{1}^{\dagger} \Sigma_{1}\right]
$$

In fact, all operators that respect the type-1,2 symmetries can be expressed in terms of 4 invariant quantities, the trace and determinant of the $2 \times 2$ Hermitian operators $\mathcal{H}_{1} \equiv \Sigma_{1}^{\dagger} \Sigma_{1}$ and $\mathcal{H}_{2} \equiv \Sigma_{2}^{\dagger} \Sigma_{2}$, which can be considered the building blocks of such scalar operators. For instance

$$
\begin{aligned}
\operatorname{Tr}\left[\mathcal{H}_{1,2}^{2}\right] & =\operatorname{Tr}\left[\mathcal{H}_{1,2}\right]^{2}-2 \operatorname{Det}\left[\mathcal{H}_{1,2}\right], \\
\operatorname{Tr}\left[\mathcal{H}_{1,2} \epsilon \mathcal{H}_{1,2}^{T} \mathcal{H}_{1,2}^{T} \epsilon\right] & =-\operatorname{Tr}\left[\mathcal{H}_{1,2}\right] \operatorname{Det}\left[\mathcal{H}_{1,2}\right]
\end{aligned}
$$

and so on.

Let us now expand these 4 invariants in terms of the physical fields: the Higgs doublet $H$ and the triplet $\Phi$. Up to fourth order in the scalar fields, we obtain the following expansions

$$
\begin{aligned}
& \operatorname{Tr}\left[\Sigma_{1,2}^{\dagger} \Sigma_{1,2}\right]=2-\frac{4}{f^{2}} \operatorname{Tr}\left[\Phi^{\dagger} \Phi\right] \pm \frac{2 i}{f^{3}}\left[H^{*} \Phi H^{\dagger} \text { - h.c. }\right]-\frac{1}{f^{4}}|H|^{4} \\
& +\frac{16}{3 f^{4}} \operatorname{Tr}\left[\Phi^{\dagger} \Phi \Phi^{\dagger} \Phi\right]+\frac{16}{3 f^{4}} H^{*} \Phi \Phi^{\dagger} H^{T}+\ldots \\
& \operatorname{Det}\left[\Sigma_{1,2}^{\dagger} \Sigma_{1,2}\right]=1-\frac{4}{f^{2}} \operatorname{Tr}\left[\Phi^{\dagger} \Phi\right] \pm \frac{2 i}{f^{3}}\left[H^{*} \Phi H^{\dagger} \text { - h.c. }\right]-\frac{1}{f^{4}}|H|^{4} \\
& -\frac{8}{3 f^{4}} \operatorname{Tr}\left[\Phi^{\dagger} \Phi \Phi^{\dagger} \Phi\right]+\frac{8}{f^{4}}\left(\operatorname{Tr}\left[\Phi^{\dagger} \Phi\right]\right)^{2}+\frac{16}{3 f^{4}} H^{*} \Phi \Phi^{\dagger} H^{T}+\ldots
\end{aligned}
$$

(with the + sign for the type- 1 case and the - sign for the type-2 case). Much of this structure is in fact dictated by the global symmetries from the first terms in the expansion [although not completely: notice that the quartic couplings for $\Phi$ do differ between (43) and (44)] so that in retrospective it is not surprising that scalar loops generate operators that were already induced by fermion or gauge boson loops.

We see that, provided the coefficients in front of these operators have the appropriate sign, the triplet $\Phi$ gains a heavy mass of order $f$. Below that threshold, one can integrate out this triplet. If the only operators present are type- 1 or type- 2 , one simply gets

$$
\Phi_{i j}= \pm \frac{i}{2 f} H_{i} H_{j}+f \mathcal{O}\left(H^{4} / f^{4}\right)
$$


and substituting back in (43) or (44) we see that there is a cancellation and the Higgs quartic coupling is zero. In the presence of both type- 1 and type- 2 operators however, this is not the case. From

$$
\begin{aligned}
\lambda_{1} \mathcal{O}_{1}(\Sigma)+\lambda_{2} \mathcal{O}_{2}(\Sigma) & =\left(\lambda_{1}+\lambda_{2}\right) f^{2} \operatorname{Tr}\left[\Phi^{\dagger} \Phi\right]+\frac{i}{2}\left(\lambda_{1}-\lambda_{2}\right) f\left[H^{*} \Phi H^{\dagger}-\text { h.c. }\right] \\
& +\frac{1}{4}\left(\lambda_{1}+\lambda_{2}\right)|H|^{4}+\ldots
\end{aligned}
$$

one obtains now

$$
\Phi_{i j}= \pm \frac{\left(\lambda_{1}-\lambda_{2}\right)}{\left(\lambda_{1}+\lambda_{2}\right)} H_{i} H_{j}+\mathcal{O}\left(H^{4}\right),
$$

and substituting back in (46) one gets a quartic coupling $(\lambda / 4)|H|^{4}$ satisfying

$$
\frac{1}{\lambda}=\frac{1}{\lambda_{1}}+\frac{1}{\lambda_{2}}
$$

Once again we see how both type-1 and type-2 couplings must be present simultaneously, this time to generate a non-zero Higgs quartic coupling.

Due to the symmetry structure of the Littlest Higgs model the $\Sigma$-operator induced by scalar loops turned out to be similar to the operators induced by gauge boson loops. In other LH models this does not need to be the case. In Appendix B we give an example, based on the $S U(6) / S p(6)$ coset structure, in which scalar loops generate genuinely new operators. Another example is the "Simplest" Little Higgs model of ref. [2] in which fermion and gauge boson loops do not induce any scalar operators. These have to be introduced by hand and once this is done they do get renormalized by scalar loops.

\section{Implications}

Consider a simple tree-level scalar potential for the Littlest Higgs of the form

$$
V_{0} / f^{4}=\kappa_{1} \operatorname{Tr}\left[\Sigma_{1}^{\dagger} \Sigma_{1}\right]+\kappa_{2} \operatorname{Tr}\left[\Sigma_{2}^{\dagger} \Sigma_{2}\right]+\kappa_{1}^{\prime} \operatorname{Det}\left[\Sigma_{1}^{\dagger} \Sigma_{1}\right]+\kappa_{2}^{\prime} \operatorname{Det}\left[\Sigma_{2}^{\dagger} \Sigma_{2}\right]
$$

In principle, operators of higher order in $\Sigma_{1,2}$ could be added but for our purposes it will be enough to keep these four. The constants $\kappa_{1,2}$ and $\kappa_{1,2}^{\prime}$ are unknown (they depend on the UV completion of the model). One can nevertheless estimate their typical size simply by looking at the radiative contributions they receive from loops of light particles. The most important correction comes from the quadratically divergent 
piece $\Lambda^{2} \operatorname{Str} M^{2} /\left(32 \pi^{2}\right)$. In this way, and using the technique explained in sect. 2 we obtain

$$
\begin{aligned}
\delta \kappa_{1} & =-\left[\frac{3}{16}\left(g_{2}^{2}+g_{2}^{\prime 2}\right)+12 \kappa_{1}-4 \kappa_{1}^{\prime}\right] \frac{\Lambda^{2}}{(4 \pi f)^{2}}, \\
\delta \kappa_{2} & =-\left[\frac{3}{16}\left(g_{1}^{2}+g_{1}^{\prime 2}\right)+12 \kappa_{2}-4 \kappa_{2}^{\prime}\right] \frac{\Lambda^{2}}{(4 \pi f)^{2}}, \\
\delta \kappa_{1}^{\prime} & =-\left[6 h_{1}^{2}+20 \kappa_{1}^{\prime}\right] \frac{\Lambda^{2}}{(4 \pi f)^{2}}, \\
\delta \kappa_{2}^{\prime} & =-20 \kappa_{2}^{\prime} \frac{\Lambda^{2}}{(4 \pi f)^{2}},
\end{aligned}
$$

and here one can substitute $\Lambda=4 \pi f$ for the NDA estimate. The interest of this calculation is the following. Note that it would not be natural to expect that the unknown constants $\kappa_{1,2}$ and $\kappa_{1,2}^{\prime}$ take numerical values much smaller than the oneloop contributions displayed in (50). Concerning the gauge boson and fermion loop contributions, they can already cause problems with the naturalness of electroweak breaking in these models [9]: phenomenology often requires values for $\kappa_{1,2}$ and $\kappa_{1,2}^{\prime}$ which are indeed significantly smaller than such one-loop corrections.

Eq. (50) includes also the new contributions from scalar loops, which have not been obtained in the literature before and have important implications. As is clear from (50) this scalar contribution is even more problematic than the rest: no matter what treelevel value for $\kappa_{1,2}$ and $\kappa_{1,2}^{\prime}$ one starts with (provided it is not zero), loop contributions tend to generate a value which is one order of magnitude larger. The problem with this is not simply that one-loop corrections are larger than the tree level result (this does not mean necessarily that the perturbative expansion is breaking down). For instance, one-loop corrections might involve some coupling that is significantly larger than the couplings entering the tree-level result so that they overcome the loop suppression (the corrections to the Higgs mass in the MSSM [13] are a famous example). The problem with (50) is that one-loop corrections involve precisely the same coupling entering at tree-level. In other words, these scalar couplings get multiplicatively renormalized as

$$
\kappa \rightarrow \kappa\left[1-\mathcal{N} \frac{\Lambda^{2}}{(4 \pi f)^{2}}\right],
$$

with large $\mathcal{N}(\mathcal{N}=12$ or 20 in this particular model $)$, so that perturbative calculability is really lost. 
The root of this pathological behaviour is the NDA substitution $\Lambda=4 \pi f$, and we are thus lead to conclude that the self-consistency of the whole non-linear sigma model approach requires a lower cutoff, satisfying at least

$$
\Lambda \lesssim \frac{4 \pi f}{\sqrt{20}},
$$

which, furthermore, probably cannot be saturated. This is in agreement with general results in technicolor theories: for instance, ref. [14] shows that the chiral perturbation theory that describes the low-energy behaviour of technicolor theories breaks down at $4 \pi f / \sqrt{\mathcal{N}_{t f}}$, where $\mathcal{N}_{t f}$ is the number of technifermions. Notice however that the result (52) arises from purely low-energy considerations about the consistency of the effective theory approach without reference to the possible UV completion of the LH model.

If we interpret this UV cutoff as the scale at which the theory becomes strongly interacting (usually taken to be $4 \pi f$ instead) then, the implication for model building of the stricter upper bound (52) is that it is not possible to keep $\Lambda=10 \mathrm{TeV}$ and $f=1 \mathrm{TeV}$. In fact, if for naturalness one keeps $f=1 \mathrm{TeV}$, the hierarchy one would be able to explain with this kind of Little Higgs theories will be much milder: $\Lambda \simeq 3$ $\mathrm{TeV}$. Conversely, if one insists in keeping $\Lambda \simeq 10 \mathrm{TeV}$, the scale $f$ is not stable at 1 $\mathrm{TeV}$ but rather pushed up to $f \simeq 3.5 \mathrm{TeV}$. While this can in fact be welcome to avoid problems with electroweak precision tests [16,17], it would generically be a disaster for the naturalness of electroweak breaking [9].

A different possibility is that the scale $4 \pi f / \sqrt{20}$ signals the appearance of new degrees of freedom/resonances (besides the pseudo-Goldstones included already in the low-energy effective theory) lighter than the scale of strong dynamics at $4 \pi f$. Such possibility can in fact be quite generic and has been discussed previously in the context of Little Higgs models [18]. In this respect, our argument in favor of such new states is purely bottom-up, with no reference to a particular UV completion but rather coming from the consistency of the low-energy effective theory. In this sense our results are complementary to unitarity arguments [19] which also point towards a cutoff scale lower than the NDA estimate. Typically, our bounds on $\Lambda$ are somewhat stronger than those coming from unitarity [e.g. for the $S U(5) / S O(5)$ model we get $\Lambda \lesssim 4 \pi f / \sqrt{20} \simeq 2.81 f$ vs. the unitarity bound $\Lambda_{U} \lesssim 3.17 f$ or $\Lambda \lesssim 4 \pi f / \sqrt{24} \simeq 2.57 f$ vs. $\Lambda_{U} \lesssim 3.68 f$ for the $S U(6) / S p(6)$ model [19]].

The size of the factor $\mathcal{N}$ seems to grow with the size of the global groups in- 
volved. In Appendix $\mathrm{C}$ we have performed the exercise of calculating this bound for a $S U(N) / S O(N)$ non-linear sigma model to see how $\mathcal{N}$ scales with $N$ finding $\mathcal{N}=4 N$. This seems then to favour LH models with small group structures. We have analyzed as a further example the "Simplest" LH model [2], based on $[S U(3) \times U(1)]^{2} /[S U(2) \times$ $U(1)]^{2}$, using the same techniques finding

$$
\Lambda \lesssim \frac{4 \pi f_{1} f_{2}}{\sqrt{3} f} \leq \frac{4 \pi f}{2 \sqrt{3}}
$$

where $f_{1}$ and $f_{2}$ are the two order parameters of the spontaneous symmetry breaking in this model, satisfying $f_{1}^{2}+f_{2}^{2}=f^{2}$. The second inequality follows from this constraint and we therefore arrive at $\mathcal{N} \geq 12$, which is indeed smaller than for larger groups but still significant.

Before moving on to other implications we should emphasize that the bounds on the cutoff we have derived by looking at the quadratically divergent corrections induced by scalar couplings are independent of how we choose to parametrize the scalar operators. For instance, instead of using the operators $\left\{\operatorname{Tr}\left[\Sigma_{1}^{\dagger} \Sigma_{1}\right], \operatorname{Tr}\left[\Sigma_{2}^{\dagger} \Sigma_{2}\right], \operatorname{Det}\left[\Sigma_{1}^{\dagger} \Sigma_{1}\right], \operatorname{Det}\left[\Sigma_{2}^{\dagger} \Sigma_{2}\right]\right\}$ we could have chosen any other linear combination of them (provided the new four operators are independent). In general, starting with a tree-level potential

$$
V_{0} / f^{4}=\sum_{\alpha=1}^{n} \kappa_{\alpha} \mathcal{O}_{\alpha}(\Sigma),
$$

we could change to a new basis of operators $\left\{\mathcal{O}_{\alpha}^{\prime}(\Sigma)\right\}$ with

$$
\mathcal{O}_{\alpha}^{\prime}(\Sigma)=A_{\alpha \beta} \mathcal{O}_{\beta}(\Sigma)
$$

where the matrix $A$ is non-singular (we are assuming repeated indices are summed over). In this new basis the tree-level potential is

$$
V_{0} / f^{4}=\sum_{\alpha=1}^{n} \kappa_{\alpha}^{\prime} \mathcal{O}_{\alpha}^{\prime}(\Sigma),
$$

with

$$
\kappa_{\alpha}^{\prime}=\kappa_{\beta} A_{\beta \alpha}^{-1}
$$

In the original basis, the quadratically divergent correction induced by these scalar operators takes the form

$$
\delta V / f^{4}=-\frac{\Lambda^{2}}{(4 \pi f)^{2}} \kappa_{\alpha} R_{\alpha \beta} \mathcal{O}_{\beta}(\Sigma),
$$


where $R$ is a numerical matrix (not normal in general). In the new basis the form of $\delta V / f^{4}$ is the same with primed quantities and the new matrix $R^{\prime}$ is related to the original $R$ by a similarity transformation:

$$
R_{\alpha \beta}^{\prime}=A_{\alpha \gamma}^{-1} R_{\gamma \rho} A_{\rho \beta}
$$

Notice that we have implicitly assumed that the bases are complete, in the sense that no new operators appear in $\delta V$ that were not present already in $V_{0}$. We also assume that $R$ is of rank $n$. If this were not the case and $\operatorname{Rank}(R)=n^{\prime}<n$ then we could have started with a smaller basis with just $n^{\prime}$ operators. Therefore, the matrix $R$ can be diagonalized and concerning the bound on the cutoff scale $\Lambda$ we are interested in its largest eigenvalue (in absolute value). It is clear then from the above discussion that the bound does not depend on what basis is chosen for the analysis because a similarity transformation like (59) leaves the eigenvalues of $R$ invariant.

In the Littlest Higgs model, the matrix $R$ can be read off directly from eq. (50):

$$
R=4\left(\begin{array}{cccc}
3 & 0 & -1 & 0 \\
0 & 3 & 0 & -1 \\
0 & 0 & 5 & 0 \\
0 & 0 & 0 & 5
\end{array}\right)
$$

and has the eigenvalues $\{12,12,20,20\}$ so that the bound on $\Lambda$ is indeed $\Lambda \lesssim 4 \pi f / \sqrt{20}$.

Finally, the new scalar contributions we have discussed might have implications for the important issue of vacuum alignment [20,21]. Expanding in physical fields the scalar potential (49), with couplings corrected by one-loop quadratic divergences as given in (50), we get

$$
\begin{aligned}
V & =4 f^{2} \operatorname{Tr}\left[\Phi^{\dagger} \Phi\right]\left\{-\left(\kappa_{1}+\kappa_{2}+\kappa_{1}^{\prime}+\kappa_{2}^{\prime}\right)\right. \\
& \left.+\frac{\Lambda^{2}}{(4 \pi f)^{2}}\left[\frac{3}{16}\left(g_{1}^{2}+g_{2}^{2}+g_{1}^{\prime 2}+g_{2}^{\prime 2}\right)+6 h_{1}^{2}+12\left(\kappa_{1}+\kappa_{2}\right)+16\left(\kappa_{1}^{\prime}+\kappa_{2}^{\prime}\right)\right]\right\}+\ldots
\end{aligned}
$$

It is easy to choose the $\kappa_{i}$ and $\kappa_{i}^{\prime}$ couplings in such a way that the mass of the triplet $\Phi$ is positive, for instance by choosing them all negative. Take into account that $\Lambda$ is bounded precisely in such a way that the scalar one-loop divergent piece cannot overcome the tree level contribution. It might seem that this last fact precludes the scalar radiative correction from having any effect in vacuum alignment. This is not so. For instance, assume that gauge and fermion couplings are negligible compared with 
the scalar $\kappa_{i}$ and $\kappa_{i}^{\prime}$ couplings so that

$$
V \simeq-4 f^{2} \operatorname{Tr}\left[\Phi^{\dagger} \Phi\right]\left\{\left(\kappa_{1}+\kappa_{2}\right)\left[1-12 \frac{\Lambda^{2}}{(4 \pi f)^{2}}\right]+\left(\kappa_{1}^{\prime}+\kappa_{2}^{\prime}\right)\left[1-16 \frac{\Lambda^{2}}{(4 \pi f)^{2}}\right]\right\}+\ldots
$$

Next take $\kappa_{1}+\kappa_{2}>0$ and $\kappa_{1}^{\prime}+\kappa_{2}^{\prime}<0$ but with $\kappa_{1}+\kappa_{2}+\kappa_{1}^{\prime}+\kappa_{2}^{\prime}<0$ (so that the chosen vacuum is stable at tree level). Scalar loop corrections in (62) tend to reduce in absolute value the $\kappa_{1}+\kappa_{2}$ and $\kappa_{1}^{\prime}+\kappa_{2}^{\prime}$ contributions but the effect is stronger for this latter (positive) piece. It is then possible that for some $\Lambda$ (well below the upper bound $4 \pi f / \sqrt{20}$ ) the negative contribution from $\kappa_{1}+\kappa_{2}$ dominates and destabilizes the vacuum. In any case, such possibilities and choices of couplings should be discussed in the context of a UV complete model.

\section{Conclusions}

Little Higgs models protect the mass of the Higgs boson from one-loop quadratically divergent corrections by making it a pseudo-Goldstone of several global symmetries broken in a collective way at some scale $f \sim 1 \mathrm{TeV}$. The models try to stabilize in this manner the little hierarchy between the electroweak scale and the $10 \mathrm{TeV}$ scale, where new physics (presumably strongly coupled) appears. Although it is not clear whether a fully satisfactory model exists (the models in the literature are either more finetuned than naively thought [9] or have problems with precision electroweak tests $[16,17]$ or both) the idea is interesting in principle.

These models predict the existence of a set of new particles with masses of order $f$ which fill out multiplets of the global symmetries together with the SM particles and are responsible for cancelling (at one loop) the dangerous quadratic divergences that affect the SM Higgs boson mass. Only this mass (or perhaps that of a second Higgs

doublets in some LH versions) is protected from one-loop quadratic divergences. The scalar potential receives the usual quadratically divergent contribution

$$
\delta V=\frac{\Lambda^{2}}{32 \pi^{2}} \operatorname{Str} M^{2}
$$

which is in fact crucial for the phenomenological viability of the models: it generates scalar operators that give masses of order $f$ to the particles beyond the SM ones introduced in these models, a Higgs quartic coupling, etc. In previous literature the contributions of gauge boson and fermion loops to the supertrace in (63) have been 
computed and discussed and the size of the resulting scalar operators is estimated using the Naive Dimensional Analysis rule $\Lambda \simeq 4 \pi f$ (which is roughly $10 \mathrm{TeV}$ for $f \simeq 1 \mathrm{TeV}$ ). These radiatively induced scalar operators are then included in the Lagrangian from the very beginning: they could clearly be present after integrating out the new physics at $\Lambda$. Once this is done there will be also a scalar contribution to the supertrace in (63) and the main purpose of this paper has been to compute this new contribution and discuss what impact it might have for the phenomenology of LH models. In calculating this new scalar contribution to the effective potential we have used with advantage a technique proposed recently in [10] which greatly simplifies the task.

We have found that, depending on the structure of global symmetries respected by the gauge sector and the fermion sector, it is possible that scalar loops generate truly new operators not induced before by loops of gauge bosons or fermions. Such is the case for some versions of the $S U(6) / S p(6) \mathrm{LH}$ model although not for the Littlest Higgs model. More importantly, we have found that in general scalar loops renormalize the existent scalar operators multiplicatively in the form

$$
\mathcal{O}_{S} \rightarrow \mathcal{O}_{S}\left[1-\mathcal{N} \frac{\Lambda^{2}}{(4 \pi f)^{2}}\right]
$$

where $\mathcal{N}$ is sizeable $[$ e.g. $\mathcal{N}=20$ in the Littlest Higgs model, $\mathcal{N}=24$ in the version of the $S U(6) / S p(6)$ model discussed in Appendix B, $\mathcal{N} \geq 12$ in the "Simplest" LH model, etc.]. Consistency of the non-linear effective theory approach demands

$$
\Lambda \lesssim \frac{4 \pi f}{\sqrt{\mathcal{N}}}
$$

which is in general significantly lower than the NDA estimate $4 \pi f \simeq 10 \mathrm{TeV}$. This can hinder the naturalness of the separation between $f \simeq 1 \mathrm{TeV}$ and $\Lambda \simeq 10 \mathrm{TeV}$, causing further difficulties in LH model building. Alternatively, it may signal the appearance of new resonances well below $10 \mathrm{TeV}$. These may be a concern for electroweak precision tests or may offer new experimental handles on LH models at the LHC.

\section{A. Log Corrections in the $S U(5) / S O(5)$ Model}

The same technique used in the main text can be applied to the calculation of the logarithmically divergent corrections to the effective potential,

$$
\delta_{L} V=-\frac{\log \Lambda^{2}}{64 \pi^{2}} \operatorname{Str} M^{4}
$$


Just for illustrative purposes, in this appendix we calculate the scalar contributions to $\delta_{L} V$ coming from the following part of the tree-level scalar potential of eq. (49)

$$
\delta V_{0} / f^{4}=\kappa_{1} \operatorname{Tr}\left[\Sigma_{1}^{\dagger} \Sigma_{1}\right]+\kappa_{2} \operatorname{Tr}\left[\Sigma_{2}^{\dagger} \Sigma_{2}\right]
$$

i.e. we will write down the corrections proportional to $\kappa_{1}^{2}, \kappa_{2}^{2}$ and $\kappa_{1} \kappa_{2}$. Because the latter corrections break both global $S U(3)_{i}$ symmetries (putting in communication sectors with different types of global symmetries) the operators proportional to $\kappa_{1} \kappa_{2}$ generated in this way cannot be expressed in terms of $\Sigma_{1}$ and $\Sigma_{2}$ only. It is then convenient to split these matrices in the following way:

$$
\Sigma_{1}=\left(\begin{array}{c}
\Theta \\
\sigma_{1}
\end{array}\right), \quad \Sigma_{2}=\left(\begin{array}{c}
\sigma_{2}^{T} \\
\Theta^{T}
\end{array}\right),
$$

where $\Theta$ is a $2 \times 2$ matrix and $\sigma_{i}$ are 2-dimensional vectors. Under $S U(2)_{1} \times S U(2)_{2}$ gauge transformations, with matrices $V_{1}$ and $V_{2}$, these quantities change as

$$
\begin{aligned}
\Theta & \rightarrow V_{1} \Theta V_{2}^{T}, \\
\sigma_{1} & \rightarrow \sigma_{1} V_{2}^{T}, \\
\sigma_{2} & \rightarrow V_{1} \sigma_{2} .
\end{aligned}
$$

Using the previous decomposition one gets

$$
\begin{aligned}
\delta_{L(1,2)} V & =-\frac{\kappa_{1} \kappa_{2}}{4 \pi^{2}} f^{4} \log \Lambda^{2}\left\{8+2 \operatorname{Tr}\left[\Sigma_{1}^{\dagger} \Sigma_{1}\right] \operatorname{Tr}\left[\Sigma_{2}^{\dagger} \Sigma_{2}\right]-4\left(\operatorname{Tr}\left[\Sigma_{1}^{\dagger} \Sigma_{1}\right]+\operatorname{Tr}\left[\Sigma_{2}^{\dagger} \Sigma_{2}\right]\right)\right. \\
& +2 \operatorname{Tr}\left[\Theta^{\dagger} \Theta\right]^{2}+9 \operatorname{Tr}\left[\Theta^{\dagger} \Theta \Theta^{\dagger} \Theta\right]-7 \operatorname{Tr}\left[\Theta^{\dagger} \Theta\right]+7\left(\sigma_{1} \Theta^{\dagger} \Theta \sigma_{1}^{\dagger}+\sigma_{2}^{\dagger} \Theta \Theta^{\dagger} \sigma_{2}\right) \\
& \left.+\frac{7}{2}\left(\Sigma_{33}^{*} \sigma_{1} \Theta^{\dagger} \sigma_{2}+\text { h.c. }\right)\right\} .
\end{aligned}
$$

One can check, from (A.4) and the fact that $\Sigma_{33}$ is an $S U(2)_{1} \times S U(2)_{2}$ singlet, that all these operators are gauge-invariant. As expected, an expansion of this correction in powers of the physical fields shows a log-divergent contribution to the mass of the Higgs boson.

For comparison, we also write down the corrections proportional to $\kappa_{a}^{2}$, which do not provide such a contribution to the Higgs mass:

$$
\delta_{L(a)} V=-\frac{\kappa_{a}^{2}}{8 \pi^{2}} f^{4} \log \Lambda^{2}\left\{4 \operatorname{Tr}\left[\Sigma_{a}^{\dagger} \Sigma_{a}\right]^{2}+9 \operatorname{Tr}\left[\Sigma_{a}^{\dagger} \Sigma_{a} \Sigma_{a}^{\dagger} \Sigma_{a}\right]-17 \operatorname{Tr}\left[\Sigma_{a}^{\dagger} \Sigma_{a}\right]\right\},
$$

with $a=1,2$. In a similar way, one could compute the logarithmically divergent corrections coming from fermion, gauge boson and other scalar loops. 


\section{B. Scalar Loops in the $S U(6) / S p(6)$ Model}

This model [3] is based on an $S U(6) / S p(6)$ nonlinear sigma model, with the spontaneous breaking of $S U(6)$ down to $S p(6)$ produced by the vacuum expectation value of a $6 \times 6$ antisymmetric matrix field $\Phi$. We follow [22] and choose

$$
\langle\Phi\rangle \equiv \Sigma_{0}=\left(\begin{array}{llll} 
& I_{2} & & \\
-I_{2} & & & \\
& & & 1 \\
& & -1 &
\end{array}\right)
$$

This breaking of the global $S U(6)$ symmetry produces 14 Goldstone bosons which include the Higgs doublet field. As usual, these Goldstone bosons can be parametrized through the nonlinear sigma model field

$$
\Sigma=e^{i \Pi / f} \Sigma_{0} e^{i \Pi^{T} / f}
$$

with $\Pi=\sum_{a} \pi^{a} X^{a}$, where $\pi_{a}$ are the Goldstone boson fields and $X^{a}$ the broken $S U(6)$ generators. The model assumes a gauged $S U(2)_{1} \times S U(2)_{2}$ subgroup of $S U(6)$ with generators ( $\sigma^{a}$ are the Pauli matrices)

$$
Q_{1}^{a}=\left(\begin{array}{ccc}
\sigma^{a} / 2 & & \\
& 0_{2} & \\
& & 0_{2}
\end{array}\right), \quad Q_{2}^{a}=\left(\begin{array}{ccc}
0_{2} & \\
& -\sigma_{2}^{a *} / 2 & \\
& & 0_{2}
\end{array}\right) .
$$

As usual, hypercharge can be embedded in different ways without destabilizing the Higgs mass due to the smallness of the $g^{\prime}$ coupling. We focus therefore only in the $S U(2)$ part of the gauge sector. The vacuum expectation value in eq. (B.1) breaks $S U(2)_{1} \times S U(2)_{2}$ down to the diagonal $S U(2)$, identified with the SM $S U(2)_{L}$ group.

The Goldstone and (pseudo)-Goldstone bosons in the hermitian matrix $\Pi$ in $\Sigma$ fall in representations of the SM group as

$$
\Pi=\frac{1}{\sqrt{2}}\left(\begin{array}{cccc}
\xi \sqrt{2} & \varphi & H_{2}^{\dagger} & H_{1}^{\dagger} \\
\varphi^{\dagger} & \xi^{*} \sqrt{2} & -H_{1}^{T} & H_{2}^{T} \\
H_{2} & -H_{1}^{*} & 0 & 0 \\
H_{1} & H_{2}^{*} & 0 & 0
\end{array}\right)+\frac{1}{\sqrt{12}} \zeta^{0} \operatorname{diag}(1,1,1,1,-2,-2)
$$

where $H_{1}=\left(h_{1}^{-}, h_{1}^{0}\right)$ and $H_{2}=\left(h_{2}^{0}, h_{2}^{+}\right)$are Higgs doublets; $\varphi$ is an antisymmetric $2 \times 2$ matrix

$$
\varphi=\left[\begin{array}{cc}
0 & \phi^{0} \\
-\phi^{0} & 0
\end{array}\right]
$$


containing a singlet $\phi^{0}$. Then, $\zeta^{0}$ is a Goldstone that might be eaten to give mass to a heavy $U(1)$ field or remain in the physical spectrum, depending on the embedding of hypercharge, and $\xi$ is the real triplet of Goldstone bosons associated to $S U(2)_{1} \times$ $S U(2)_{2} \rightarrow S U(2)$ breaking:

$$
\xi=\frac{1}{2} \sigma^{a} \xi^{a}=\left[\begin{array}{cc}
\frac{1}{2} \xi^{0} & \frac{1}{\sqrt{2}} \xi^{+} \\
\frac{1}{\sqrt{2}} \xi^{-} & -\frac{1}{2} \xi^{0}
\end{array}\right] .
$$

All the fields in $\Pi$ as written above are canonically normalized.

The kinetic part of the Lagrangian is

$$
\mathcal{L}_{\text {kin }}=\frac{f^{2}}{8} \operatorname{Tr}\left[\left(D_{\mu} \Sigma\right)\left(D^{\mu} \Sigma\right)^{\dagger}\right],
$$

where

$$
D_{\mu} \Sigma=\partial_{\mu} \Sigma-i \sum_{j=1}^{2} g_{j} W_{j \mu}^{a}\left(Q_{j}^{a} \Sigma+\Sigma Q_{j}^{a T}\right),
$$

with an additional $U(1)$ contribution that we neglect.

In this model, the additional fermions required to cancel the top quadratic divergences can be introduced in several ways $[3,22,5]$. We consider here the fermionic couplings chosen in [5], which are quite similar to the $S U(5) / S O(5)$ choice described in the main text. Again there is a coloured pair of new fermions $t^{\prime}, t^{\prime c}$ and the relevant part of the Lagrangian containing the top Yukawa coupling is given by

$$
\mathcal{L}_{f}=\frac{1}{2} h_{1} f \epsilon_{i j k} \epsilon_{x y} \chi_{i} \Sigma_{j x} \Sigma_{k y} u_{3}^{\prime c}+h_{2} f t^{\prime} t^{\prime c}+\text { h.c. },
$$

where now $\chi_{i}=\left(t, b, 0,0,0, t^{\prime}\right)$, the indices $i, j, k$ run through $\{1,2,6\}$ and $x, y$ from 3 to 4 , and $\epsilon_{i j k}$ and $\epsilon_{x y}$ are the completely antisymmetric tensors of dimension 3 and 2, respectively.

As in the $S U(5) / S O(5)$ Little Higgs model, gauge and fermion loops induce operators in the scalar potential of the form,

$$
\begin{aligned}
& \mathcal{O}_{V_{i}}(\Sigma)=f^{4} g_{i}^{2} \sum_{a} \operatorname{Tr}\left[\left(Q_{i}^{a} \Sigma\right)\left(Q_{i}^{a} \Sigma\right)^{*}\right] \\
& \mathcal{O}_{F}(\Sigma)=-3 f^{4} h_{1}^{2} \epsilon^{w x} \epsilon_{y z} \Sigma_{i w} \Sigma_{j x} \Sigma^{i y *} \Sigma^{j z *} .
\end{aligned}
$$

It is easy to show that

$$
\begin{aligned}
\sum_{a} \operatorname{Tr}\left[\left(Q_{1}^{a} \Sigma\right)\left(Q_{1}^{a} \Sigma\right)^{*}\right] & =\frac{3}{2}-\frac{3}{4} \operatorname{Tr}\left[\Sigma_{2}^{\dagger} \Sigma_{2}\right], \\
\sum_{a} \operatorname{Tr}\left[\left(Q_{2}^{a} \Sigma\right)\left(Q_{2}^{a} \Sigma\right)^{*}\right] & =\frac{3}{2}-\frac{3}{4} \operatorname{Tr}\left[\Sigma_{1}^{\dagger} \Sigma_{1}\right],
\end{aligned}
$$


where now

$$
\Sigma_{1}=\left(\Sigma_{i^{\prime} x^{\prime}}\right), \quad \Sigma_{2}=\left(\Sigma_{i^{\prime \prime} x^{\prime \prime}}\right) .
$$

with $i^{\prime}=\{1,2,5,6\}$ and $x^{\prime}=\{3,4\} ; i^{\prime \prime}=\{3,4,5,6\}$, and $x^{\prime \prime}=\{1,2\}$; and

$$
\epsilon^{w x} \epsilon_{y z} \Sigma_{i w} \Sigma_{j x} \Sigma^{i y *} \Sigma^{j z *}=2 \operatorname{Det}\left[\Sigma_{3}^{\dagger} \Sigma_{3}\right]
$$

with

$$
\Sigma_{3}=\left(\Sigma_{i x}\right)
$$

and $i=\{1,2,6\}, x=\{3,4\}$.

Once these scalar operators are added to the Lagrangian we should also consider the contribution of scalar loops to the quadratic divergence of the effective potential. As in the $S U(5) / S O(5)$ model, the fermion-induced operator $\mathcal{O}_{F}(\Sigma)$ generates in this way a scalar operator

$$
\mathcal{O}_{S}(\Sigma) \equiv \operatorname{Tr}\left[\Sigma_{3}^{\dagger} \Sigma_{3}\right]
$$

which in this particular case was not induced already by gauge-boson loops.

The reason behind this behaviour is of course that unlike what happened in the Littlest Higgs model, the symmetry properties of the fermion couplings in the Lagrangian are not those of the gauge boson sector. More explicitly, type-2 operators like (B.12) are invariant under a global $S U(4)_{2}$ symmetry and type-1 operators like (B.13) are invariant under a different global $S U(4)_{1}$ symmetry. The transformation properties of $\Sigma_{1,2}$ are

$$
\Sigma_{1} \rightarrow U_{1} \Sigma_{1} V_{2}^{T}, \quad \Sigma_{2} \rightarrow U_{2} \Sigma_{1} V_{1}^{T}
$$

with $U_{i}$ a (global) $S U(4)_{i}$ matrix and $V_{i}$ a (local) $S U(2)_{i}$ matrix. The global $S U(4)_{i}$ symmetries guarantee the lightness of both Higgs doublets $H_{1}$ and $H_{2}$. The operators (B.15) and (B.17) are not of type-1 or type-2 but rather of a different type- 3 with $\Sigma_{3}$ transforming as

$$
\Sigma_{3} \rightarrow U_{3} \Sigma_{1} V_{2}^{T}
$$

with $U_{3}$ a matrix of a (global) $S U(3)_{1}$ which is a subgroup of $S U(4)_{1}$. This $S U(3)_{1}$ does not keep $H_{2}$ light and therefore, in the presence of type-3 operators, only $H_{1}$ will remain at the electroweak scale.

Writing the tree-level potential as

$$
V_{0} / f^{4}=\kappa_{1} \operatorname{Tr}\left[\Sigma_{1}^{\dagger} \Sigma_{1}\right]+\kappa_{2} \operatorname{Tr}\left[\Sigma_{2}^{\dagger} \Sigma_{2}\right]+\kappa_{3} \operatorname{Tr}\left[\Sigma_{3}^{\dagger} \Sigma_{3}\right]+\kappa_{3}^{\prime} \operatorname{Det}\left[\Sigma_{3}^{\dagger} \Sigma_{3}\right]
$$


we can compute the one-loop quadratically divergent contributions to this potential from loops of gauge bosons [neglecting again $U(1)$ corrections], fermions and scalars. We obtain

$$
\begin{aligned}
\delta V / f^{4} & =-\frac{\Lambda^{2}}{(4 \pi f)^{2}}\left\{\left(\frac{9}{16} g_{2}^{2}+10 \kappa_{1}\right) \operatorname{Tr}\left[\Sigma_{1}^{\dagger} \Sigma_{1}\right]+\left(\frac{9}{16} g_{1}^{2}+10 \kappa_{2}\right) \operatorname{Tr}\left[\Sigma_{2}^{\dagger} \Sigma_{2}\right]\right. \\
& +\left(10 \kappa_{3}-8 \kappa_{3}^{\prime}\right) \operatorname{Tr}\left[\Sigma_{3}^{\dagger} \Sigma_{3}\right]+4 \kappa_{3}^{\prime} \operatorname{Tr}\left[\Sigma_{3}^{\dagger} \Sigma_{3} \Sigma_{3}^{\dagger} \Sigma_{3}\right]+\left(6 h_{1}^{2}+16 \kappa_{3}^{\prime}\right) \operatorname{Det}\left[\Sigma_{3}^{\dagger} \Sigma_{3}\right] \\
& \left.+4 \kappa_{3}^{\prime} \sigma_{5} \Sigma_{3}^{\dagger} \Sigma_{3} \sigma_{5}^{\dagger}+4 \kappa_{3}^{\prime} d_{5}^{\dagger} \Sigma_{3} \Sigma_{3}^{\dagger} d_{5}\right\},
\end{aligned}
$$

where $d_{5} \equiv\left(\Sigma_{i 5}\right)$ and $\sigma_{5} \equiv\left(\Sigma_{5 x}\right)$ (with $i=\{1,2,6\}$ and $\left.x=\{3,4\}\right)$. We see that new operators not present in (B.21) are generated and one should also include them from the beginning for a complete analysis. For such complete analysis we should include the operators $^{2} T_{\alpha} \equiv \operatorname{Tr}\left[\Sigma_{\alpha}^{\dagger} \Sigma_{\alpha}\right](\alpha=1,3), \operatorname{Tr}\left[\Sigma_{3}^{\dagger} \Sigma_{3} \Sigma_{3}^{\dagger} \Sigma_{3}\right], T_{3}^{2}, d_{5}^{\dagger} \Sigma_{3} \Sigma_{3}^{\dagger} d_{5}, \sigma_{5} \Sigma_{3}^{\dagger} \Sigma_{3} \sigma_{5}^{\dagger}$, $d_{5}^{\dagger} d_{5}, \sigma_{5} \sigma_{5}^{\dagger}, d_{5}^{\dagger} d_{5} T_{3}$ and $\sigma_{5} \sigma_{5}^{\dagger} T_{3}$. Using the previous basis, the matrix $R$ of eq. (58) is 11-dimensional and breaks up in two blocks. The first $2 \times 2$ block, in the space spanned by $\left\{T_{1}, T_{2}\right\}$, is simply $-10 I_{2}$. The $9 \times 9$ block is

$$
R=\left(\begin{array}{ccccccccc}
10 & 0 & 0 & 0 & 0 & 0 & 0 & 0 & 0 \\
-16 & 16 & 4 & -4 & -4 & 0 & 0 & 0 & 0 \\
-32 & 8 & 20 & 4 & 4 & 0 & 0 & 0 & 0 \\
-2 & 0 & 0 & 20 & 4 & -4 & 0 & 4 & 0 \\
-2 & 0 & 0 & 4 & 20 & 0 & -6 & 0 & 4 \\
0 & 0 & 0 & 0 & 0 & 10 & 0 & 0 & 0 \\
0 & 0 & 0 & 0 & 0 & 0 & 10 & 0 & 0 \\
-6 & 0 & 0 & 4 & -4 & -12 & 0 & 20 & 0 \\
-4 & 0 & 0 & -4 & 4 & 0 & -12 & 0 & 20
\end{array}\right)
$$

and has eigenvalues $\{10(3), 24(3), 12(2), 20\}$ (with the multiplicities given in parenthesis). We can then deduce the bound

$$
\Lambda \lesssim 4 \pi f / \sqrt{24}
$$

Concerning vacuum alignment issues [20,21], an expansion of the scalar potential (B.20) including the corrections in (B.21) gives

$$
\begin{aligned}
V & =4 f^{2}\left|\phi^{0}\right|^{2}\left\{\kappa_{1}+\kappa_{2}-\left(\kappa_{3}+\kappa_{3}^{\prime}\right)\right. \\
& \left.+\frac{\Lambda^{2}}{(4 \pi f)^{2}}\left[-\frac{9}{16}\left(g_{1}^{2}+g_{2}^{2}\right)+6 h_{1}^{2}-10\left(\kappa_{1}+\kappa_{2}\right)+10 \kappa_{3}+16 \kappa_{3}^{\prime}\right]\right\} \\
& +2 f^{2}\left|H_{2}\right|^{2}\left\{-\left(\kappa_{3}+\kappa_{3}^{\prime}\right)+\frac{\Lambda^{2}}{(4 \pi f)^{2}}\left[6 h_{1}^{2}+10 \kappa_{3}+12 \kappa_{3}^{\prime}\right]\right\}+\ldots
\end{aligned}
$$

\footnotetext{
${ }^{2}$ We have used the relation $\operatorname{Det}\left[\Sigma_{3}^{\dagger} \Sigma_{3}\right]=\left(T_{3}^{2}-\operatorname{Tr}\left[\Sigma_{3}^{\dagger} \Sigma_{3} \Sigma_{3}^{\dagger} \Sigma_{3}\right]\right) / 2$.
} 
It is then easy to choose the unknown couplings $\kappa_{\alpha}$ and $\kappa_{3}^{\prime}$ to overcome the negative correction from gauge boson loops (a known problem of this LH model) in such a way that both $\phi^{0}$ and $H_{2}$ have positive masses (of order $f$ ). Justifying such choice of couplings is only possible in the context of a UV completion of the model.

\section{The $S U(N) / S O(N)$ Case}

In order to gain some understanding on how the large renormalization effects from scalar loops scale with the size of the groups involved, we consider here, as a simple exercise, the case of a Little Higgs model with coset structure $S U(N) / S O(N)$. We simply repeat the loop calculation in the mean text keeping track of the $N$-dependence.

We normalize the $S U(N)$ generators as

$$
\operatorname{Tr}\left(T^{a} T^{b}\right)=\delta_{a b}
$$

We then write the well known relations (see e.g. [23])

$$
\begin{aligned}
\sum_{a \in S U(N)} T_{i j}^{a} T_{k l}^{a} & =\delta_{i l} \delta_{j k}-\frac{1}{N} \delta_{i j} \delta_{k l} \\
\sum_{a \in S O(N)} T_{i j}^{a} T_{k l}^{a} & =\frac{1}{2}\left(\delta_{i l} \delta_{j k}-\delta_{i k} \delta_{j l}\right)
\end{aligned}
$$

from which we obtain

$$
\sum_{a \in S U(N) / S O(N)} T_{i j}^{a} T_{k l}^{a} \equiv \sum_{a}\left[X_{a}^{(0)}\right]_{i j}\left[X_{a}^{(0)}\right]_{k l}=\frac{1}{2} \delta_{i l} \delta_{j k}+\frac{1}{2} \delta_{i k} \delta_{j l}-\frac{1}{N} \delta_{i j} \delta_{k l},
$$

where $a \in S U(N) / S O(N)$ runs over the broken generators, $X_{a}^{(0)}$. The superindex 0 refers to the original basis where the $\mathrm{VEV}$ producing the $S U(N) \rightarrow S O(N)$ breaking is simply the identity $I_{5}$. In that basis we then find (the diagrammatic techniques of [23] proved very useful in deriving these and similar identities)

$$
\sum_{a} \operatorname{Tr}\left[X_{a}^{(0)} Y X_{a}^{(0)} Z\right]=\sum_{a} \operatorname{Tr}\left[X_{a}^{(0)} Y X_{a}^{(0) *} Z\right]=\frac{1}{2} \operatorname{Tr}[Y] \operatorname{Tr}[Z]-\frac{1}{N} \operatorname{Tr}[Y Z]+\frac{1}{2} \operatorname{Tr}\left[Y Z^{T}\right]
$$

In a rotated basis with generators $X_{a}=U_{0} X_{a}^{(0)} U_{0}^{\dagger}$ and VEV $\Sigma_{0}=U_{0} U_{0}^{T}$ we get

$$
\begin{aligned}
\sum_{a} \operatorname{Tr}\left[X_{a} Y X_{a}^{*} Z\right] & =\frac{1}{2} \operatorname{Tr}\left[Y Z^{T}\right]-\frac{1}{N} \operatorname{Tr}[Y Z]+\frac{1}{2} \operatorname{Tr}\left[Y \Sigma_{0}^{*}\right] \operatorname{Tr}\left[Z \Sigma_{0}\right], \\
\sum_{a} \operatorname{Tr}\left[X_{a} Y X_{a} Z\right] & =\frac{1}{2} \operatorname{Tr}[Y] \operatorname{Tr}[Z]-\frac{1}{N} \operatorname{Tr}[Y Z]+\frac{1}{2} \operatorname{Tr}\left[Y \Sigma_{0} Z^{T} \Sigma_{0}^{*}\right]
\end{aligned}
$$


of which eq. (26) is a particular case with $N=5$.

Following the $S U(5) / S O(5)$ case we can gauge two $S U(2) \times U(1)$ subgroups of $\mathrm{SU}(\mathrm{N})$ so that, when $g_{1}=g_{1}^{\prime}=0$ there is a global $S U(N-2)$ symmetry protecting

the mass of the Higgs, etc. The submatrices $\Sigma_{1}$ and $\Sigma_{2}$ are generalized to $(N-2) \times 2$ matrices transforming as $\Sigma_{1} \rightarrow U_{1} \Sigma_{1} V_{2}^{T}$ and $\Sigma_{2} \rightarrow U_{2} \Sigma_{2} V_{1}^{T}$, where $U_{i}$ is a matrix of the global $S U(N-2)_{i}$ and $V_{i}$ a matrix of the local $S U(2)_{i}$. Writing down the tree-level scalar potential as in (49) we can again compute the contribution of divergent scalar loops to the same scalar operators obtaining

$$
\begin{aligned}
& \delta_{S} \kappa_{1}=-\left[2(N+1) \kappa_{1}-2(N-3) \kappa_{1}^{\prime}\right] \frac{\Lambda^{2}}{(4 \pi f)^{2}}, \\
& \delta_{S} \kappa_{2}=-\left[2(N+1) \kappa_{2}-2(N-3) \kappa_{2}^{\prime}\right] \frac{\Lambda^{2}}{(4 \pi f)^{2}}, \\
& \delta_{S} \kappa_{1}^{\prime}=-4 N \kappa_{1}^{\prime} \frac{\Lambda^{2}}{(4 \pi f)^{2}}, \\
& \delta_{S} \kappa_{2}^{\prime}=-4 N \kappa_{2}^{\prime} \frac{\Lambda^{2}}{(4 \pi f)^{2}} .
\end{aligned}
$$

From this result we conclude that the upper limit on the cut-off scale that follows from requiring consistency of the low-energy effective theory approach is

$$
\Lambda \lesssim 4 \pi f / \sqrt{4 N} .
$$

[The number of pseudo-Goldstones in this example is $\left(N^{2}+N-2\right) / 2$.]

\section{Acknowledgments}

We thank Ann Nelson for useful correspondence. This work is supported by the Spanish Ministry of Education and Science through a M.E.C. project (FPA2004-02015) and by a Comunidad de Madrid project (HEPHACOS; P-ESP-00346). José Miguel No acknowledges the financial support of a FPU grant from M.E.C.

\section{References}

[1] N. Arkani-Hamed, A. G. Cohen, E. Katz and A. E. Nelson, JHEP 0207 (2002) 034 [hep-ph/0206021].

[2] M. Schmaltz, JHEP 0408 (2004) 056 [hep-ph/0407143]. 
[3] I. Low, W. Skiba and D. Smith, Phys. Rev. D 66 (2002) 072001 [hep-ph/0207243].

[4] H. C. Cheng and I. Low, [hep-ph/0405243]; JHEP 0309 (2003) 051 [hep$\mathrm{ph} / 0308199]$.

[5] Z. Han and W. Skiba, Phys. Rev. D 72 (2005) 035005 [hep-ph/0506206].

[6] M. Schmaltz, Nucl. Phys. Proc. Suppl. 117 (2003) 40 [hep-ph/0210415]; D. E. Kaplan and M. Schmaltz, JHEP 0310 (2003) 039 [hep-ph/0302049].

[7] J. G. Wacker, [hep-ph/0208235].

[8] A. Manohar and H. Georgi, Nucl. Phys. B 234 (1984) 189; H. Georgi and L. Randall, Nucl. Phys. B 276 (1986) 241; H. Georgi, Phys. Lett. B 298 (1993) 187 [hep-ph/9207278].

[9] J. A. Casas, J. R. Espinosa and I. Hidalgo, JHEP 0503 (2005) 038 [hep$\mathrm{ph} / 0502066]$.

[10] J. R. Espinosa, M. Losada and A. Riotto, Phys. Rev. D 72 (2005) 043520 [hep$\mathrm{ph} / 0409070]$.

[11] F. Bazzocchi, M. Fabbrichesi and M. Piai, Phys. Rev. D 72 (2005) 095019 [hepph/0506175]; A. Dobado, L. Tabares and S. Penaranda, [hep-ph/0606031].

[12] S. Kamefuchi, L. O'Raifeartaigh and A. Salam, Nucl. Phys. 28, 529 (1961).

[13] J. R. Ellis, G. Ridolfi and F. Zwirner, Phys. Lett. B 262 (1991) 477; R. Barbieri, M. Frigeni and F. Caravaglios, Phys. Lett. B 258 (1991) 167; Y. Okada, M. Yamaguchi and T. Yanagida, Phys. Lett. B 262 (1991) 54; Prog. Theor. Phys. 85 (1991) 1.

[14] M. Soldate and R. Sundrum, Nucl. Phys. B 340 (1990) 1;

[15] R. S. Chivukula, M. J. Dugan and M. Golden, Phys. Rev. D 47 (1993) 2930 [hep-ph/9206222]; Phys. Lett. B 292 (1992) 435 [hep-ph/9207249].

[16] C. Csaki, J. Hubisz, G. D. Kribs, P. Meade and J. Terning, Phys. Rev. D 67 (2003) 115002 [hep-ph/0211124]; Phys. Rev. D 68 (2003) 035009 [hep-ph/0303236]. 
[17] J. L. Hewett, F. J. Petriello and T. G. Rizzo, JHEP 0310 (2003) 062 [hepph/0211218]; T. Han, H. E. Logan, B. McElrath and L. T. Wang, Phys. Rev. D 67, 095004 (2003) [hep-ph/0301040]; M. C. Chen and S. Dawson, Phys. Rev. D 70 (2004) 015003 [hep-ph/0311032]; R. Casalbuoni, A. Deandrea and M. Oertel, JHEP 0402 (2004) 032 [hep-ph/0311038]; G. Marandella, C. Schappacher and A. Strumia, Phys. Rev. D 72 (2005) 035014 [hep-ph/0502096].

[18] M. Piai, A. Pierce and J. G. Wacker, [hep-ph/0405242]; J. Thaler, JHEP 0507 (2005) 024 [hep-ph/0502175].

[19] S. Chang and H. J. He, Phys. Lett. B 586 (2004) 95 [hep-ph/0311177].

[20] M. E. Peskin, Nucl. Phys. B 175 (1980) 197.

[21] C. Cheung and J. Thaler, JHEP 0608 (2006) 016 [hep-ph/0604259];

[22] T. Gregoire, D. R. Smith and J. G. Wacker, Phys. Rev. D 69 (2004) 115008 [hep-ph/0305275].

[23] P. Cvitanović, Phys. Rev. D 14 (1976) 1536. 